Revta brasil. Bot., São Paulo, V.24, n.2, p.145-154, jun. 2001

\title{
Autocorrelação espacial das freqüências alélicas em subpopulações de cagaiteira (Eugenia dysenterica DC., Myrtaceae) no sudeste de Goiás ${ }^{1}$
}

\author{
MARIANA P. DE C. TELLES ${ }^{2,5}$, JOSÉ ALEXANDRE F. DINIZ-FILHO ${ }^{3}$, ALEXANDRE \\ S.G. COELHO ${ }^{3}$ e LÁZARO J. CHAVES ${ }^{4}$
}

(recebido: 10 de maio de 2000; aceito: 11 de janeiro de 2001)

\begin{abstract}
Spatial autocorrelation of allelic frequencies in Eugenia dysenterica DC. subpopulations (Myrtaceae) in southeastern Goiás State). Among the many fruit trees from "cerrado" region that show large economic potential in traditional agricultural systems, the "cagaiteira" (Eugenia dysenterica DC.) deserves a special place. In this work, spatial autocorrelation analyses were used to evaluate spatial patterns of genetic variation among ten local populations from southeastern Goiás State, Brazil. Six isoenzymatic markers (SKDH, 6-PGD, $\alpha$-EST, MDH, PGI e PGM) were used to evaluate genetic variability, in a total of eight polymorphic loci. Moran's I coefficients were estimated for four geographic distance classes, and correlograms thus obtained showed clinal patterns of variation for most alleles. Simulations of neutral evolution among local populations were performed using the Ornstein-Uhlenbeck process, both to establish null patterns to test this hypothesis and to define the limits of similarity among correlograms. These analyses showed that population differentiation in this species probably occurred under a neutral process in which local drift is counteracted by low geographic distance gene flow, as in isolation-by-distance or stepping-stone models.
\end{abstract}

RESUMO - (Autocorrelação espacial das freqüências alélicas em subpopulações de cagaiteira (Eugenia dysenterica DC., Myrtaceae) no sudeste de Goiás). Dentre as diversas árvores frutíferas nativas dos cerrados, a cagaiteira (Eugenia dysenterica DC.) merece destaque pelo seu amplo potencial econômico. A fim de fornecer algumas informações relativas ao padrão espacial da variabilidade genética desta espécie, foram realizadas análises de autocorrelação espacial das freqüências alélicas em dez subpopulações locais da região sudeste do Estado de Goiás. Foram utilizados marcadores isoenzimáticos, em um total de seis sistemas enzimáticos (SKDH, 6-PGD, $\alpha$-EST, MDH, PGI e PGM), com oito locos polimórficos. Foi realizada uma análise de autocorrelação espacial, utilizando índices I de Moran estimados em quatro classes de distância geográfica. Os correlogramas mostraram que, de fato, a divergência genética está estruturada no espaço em um padrão clinal de variação. Simulações de evolução neutra da variação nas frequêencias alélicas entre as subpopulações, geradas a partir de um processo Ornstein-Uhlenbeck (O-U), foram utilizadas para avaliar os padrões espaciais sob essa hipótese e compará-los com os correlogramas obtidos com as frequiências alélicas. As análises indicaram que um processo estocástico (evolução neutra) deve ser o responsável pela diferenciação genética dessas populações, havendo assim um balanço entre fluxo gênico em pequenas distâncias geográficas e deriva genética dentro das populações locais, como o esperado para modelos de isolamento-por-distância ou "stepping-stone".

Key words - Eugenia dysenterica, isozymes, neutral evolution, spatial autocorrelation

\section{Introdução}

Os estudos relativos à organização da variabilidade ou estrutura genética em populações naturais de plantas têm permitido grandes avanços no conhecimento dos processos microevolutivos envolvidos em sua diferenciação (Reis 1996). A introdução da técnica de eletroforese de isoenzimas

1. Parte da dissertação de mestrado de M.P.C. Telles.

2. Universidade Católica de Goiás, Departamento de Zootecnia, Caixa Postal 86, Av. Universitária, 1440, 74605-010 Goiânia, GO, Brasil.

3. Universidade Federal de Goiás, Instituto de Ciências Biológicas, Departamento de Biologia Geral, Caixa Postal 131, 74001-970 Goiânia, GO, Brasil.

4. Universidade Federal de Goiás, Escola de Agronomia, Caixa Postal 131, 74001-970, Goiânia, GO, Brasil.

5. Autor para correspondência: tellesmpc@uol.com.br como método de avaliação desta variabilidade revolucionou a genética de populações em meados dos anos 60 e ainda mostra potencial, apesar de outras técnicas moleculares de detecção de polimorfismo genético ao nível de DNA (RAPD, microssatélites, RFLP, AFLP, por exemplo) estarem disponíveis atualmente (Ferreira \& Grattapaglia 1998, Newton et al. 1999).

Usualmente, a análise da estrutura genética das populações está mais voltada para a caracterização dos níveis de diversidade (porcentagem de locos polimórficos, número de alelos por loco e heterozigosidade média) e para a distribuição da variabilidade genética entre e dentro das populações, que se iniciou com a análise das estatísticas-F (Wright 1951). Atualmente, outras formulações análogas são utilizadas, como a análise de variância de freqüências alélicas proposta por Cockerhan 
(1969) ou a análise de diversidade de Nei (1973). Apesar da grande importância dessas estatísticas para a caracterização genética de populações naturais, elas permitem apenas uma descrição geral da heterogeneidade espacial da variabilidade existente (Barbujani 1987).

As inferências sobre os processos microevolutivos atuando nas populações podem ser efetuadas de forma mais completa com a utilização de técnicas mais elaboradas de análise espacial (Slatkin \& Arter 1991, Epperson 1997). A análise de autocorrelação espacial (Sokal \& Oden 1978a, b, Sokal \& Oden 1991, Heywood 1991) representa uma estratégia mais geral e mais eficiente para descrever "superfícies" de variação genética complexas, avaliando a semelhança entre os valores das freqüências alélicas nas subpopulações vizinhas espacialmente e como essa semelhança se altera à medida que se modifica a escala geográfica.

Dentre as diversas árvores frutíferas nativas dos cerrados que apresentam potencial de utilização em sistemas tradicionais de produção agrícola, a cagaiteira (Eugenia dysenterica DC.), da família Myrtaceae, merece destaque pelo amplo potencial econômico (Almeida 1998). Suas populações se encontram em regiões onde predomina o clima subtropical mais fresco e vegeta em regiões de cerradões menos pobres, sendo assim uma planta perene típica nas diversas fitofisionomias da região dos cerrados brasileiros (Donadio et al. 1992). A cagaiteira possui flores hermafroditas e autocompatíveis, apresentando uma sincronia no florescimento, em um padrão de floração que é denominado "Big Bang" (Proença \& Gibbs 1994). As abelhas (incluindo Bombus sp.) são seus polinizadores preferenciais (Proença \& Gibbs 1994), apesar de existirem polinizadores oportunistas. A dispersão é realizada principalmente por macacos (primatocórico) e pelo Homem (antropocórico), embora outros animais também desempenhem esta função (Ferreira \& Cunha 1980). Essas características de história de vida, bem como os processos antrópicos e fragmentação da região do cerrado, devem ser importantes para explicar os padrões de variabilidade genética nessa espécie.

O objetivo geral deste trabalho é avaliar os padrões espaciais da variação genética para marcadores isoenzimáticos em dez subpopulações de cagaiteira (Eugenia dysenterica DC.) da região sudeste do Estado de Goiás, a fim de inferir os prováveis processos microevolutivos que estão atuando na diferenciação genética dessas subpopulações. O reconhecimento desses padrões e processos é importante já que pode fornecer subsídios para futuros programas de conservação, manejo, domesticação e melhoramento genético dessa espécie.

\section{Material e métodos}

Foram analisadas 112 progênies (famílias de irmãos oriundas de uma mesma planta-mãe, com paternidade desconhecida) coletadas em 10 subpopulações naturais de Eugenia dysenterica DC. distribuídas na região sudeste do Estado de Goiás (figura 1; tabela 1). Os 704 indivíduos provenientes dessas progênies foram submetidos à análise genética utilizando-se marcadores enzimáticos (isoenzimas), conforme protocolo previamente estabelecido (Alfenas et al. 1991). A análise dos polimorfismos enzimáticos foi realizada no Laboratório de Genética Vegetal do Departamento de Biologia Geral da UFG.

As amostras foliares das plântulas mantidas sob condições de viveiro foram submetidas à extração por maceração das folhas com bastão de vidro em almofariz de porcelana, acondicionado em cuba de gelo na presença de areia lavada e solução tampão Feret, descrita por Pitel \& Cheliak (1984), com a adição de polivinilpirrolidona (PVP). Os extratos foram submetidos à separação por eletroforese em gel de penetrose a $13,5 \%$, utilizando-se como sistema de migração o tampão Tris-histidina (pH 6,0) descrito em Lebrun \& Chevalier (1988). As fatias de gel foram então submetidas à revelação para as seguintes enzimas:

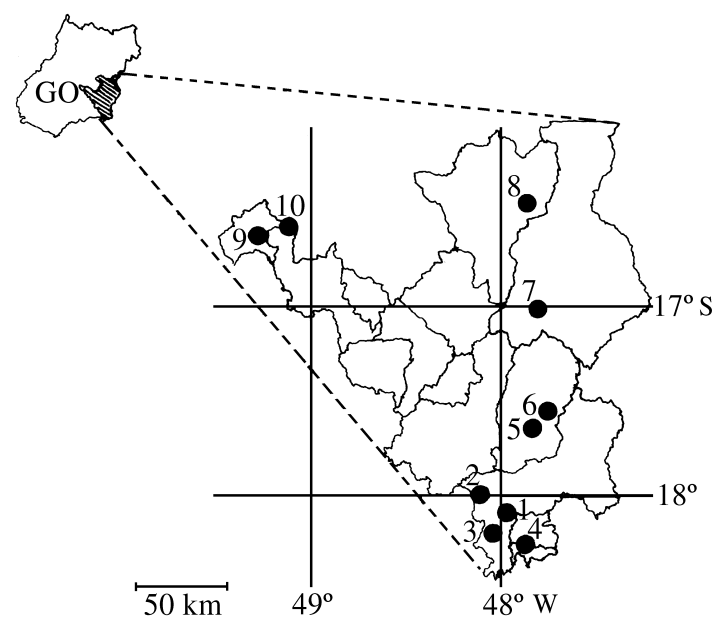

Figura 1. Municípios da região sudeste de Goiás e localização das dez áreas de coleta de cagaiteira (Eugenia dysenterica DC.), de acordo com a tabela 1 . 
Tabela 1. Localidades amostradas, coordenadas geográficas e número de árvores de cagaiteira amostradas, no sudeste de Goiás, 1996.

\begin{tabular}{|c|c|c|c|c|c|}
\hline Áreas & $\begin{array}{l}\text { Localidade } \\
\text { (município) }\end{array}$ & $\begin{array}{c}\text { Número de árvores } \\
\text { amostradas }\end{array}$ & $\begin{array}{l}\text { Altitude } \\
(\mathrm{m})\end{array}$ & $\begin{array}{l}\text { Latitude } \\
(\mathrm{S})\end{array}$ & $\begin{array}{c}\text { Longitude } \\
\text { (W) }\end{array}$ \\
\hline 1 & Catalão & 12 & 880 & $18^{\circ} 07^{\prime} 35^{\prime \prime}$ & $47^{\circ} 54^{\prime} 20^{\prime \prime}$ \\
\hline 2 & Catalão & 12 & 860 & $18^{\circ} 02^{\prime} 03^{\prime \prime}$ & $48^{\circ} 02^{\prime} 31^{\prime \prime}$ \\
\hline 3 & Catalão & 12 & 800 & $18^{\circ} 13^{\prime} 39^{\prime \prime}$ & $47^{\circ} 58^{\prime} 12^{\prime \prime}$ \\
\hline 4 & Três Ranchos & 12 & 820 & $18^{\circ} 17^{\prime} 15^{\prime \prime}$ & $47^{\circ} 48^{\prime} 41^{\prime \prime}$ \\
\hline 5 & Campo Alegre de Goiás & 12 & 930 & $17^{\circ} 39^{\prime} 11^{\prime \prime}$ & $47^{\circ} 46^{\prime} 37^{\prime \prime}$ \\
\hline 6 & Campo Alegre de Goiás & 12 & 780 & $17^{\circ} 34^{\prime} 24^{\prime \prime}$ & $47^{\circ} 42^{\prime} 12^{\prime \prime}$ \\
\hline 7 & Cristalina & 8 & 890 & $17^{\circ} 10^{\prime} 47^{\prime \prime}$ & $47^{\circ} 31^{\prime} 07^{\prime \prime}$ \\
\hline 8 & Luziânia & 12 & 900 & $16^{\circ} 28^{\prime} 48^{\prime \prime}$ & $47^{\circ} 48^{\prime} 40^{\prime \prime}$ \\
\hline 9 & Goiânia & 8 & 740 & $16^{\circ} 40^{\prime} 30^{\prime \prime}$ & $49^{\circ} 14^{\prime} 42^{\prime \prime}$ \\
\hline 10 & Senador Canedo & 12 & 840 & $16^{\circ} 37^{\prime} 13^{\prime \prime}$ & $49^{\circ} 04^{\prime} 29^{\prime \prime}$ \\
\hline
\end{tabular}

malato desidrogenase (MDH), $\alpha$-esterase ( $\alpha$-EST), chiquimato desidrogenase (SKDH), desidrogenase do ácido 6-fosfoglucônico (6-PGD), fosfoglucomutase (PGM) e fosfoglucoisomerase (PGI). Os zimogramas resultantes foram esquematizados para posterior interpretação.

A identificação e interpretação dos zimogramas foram realizadas seguindo a mesma definição dos padrões de enzimas disponíveis na literatura (Alfenas et al. 1991). Foram obtidas as frequiências alélicas e genotípicas em cada loco, considerando-se o total de plântulas analisadas em cada subpopulação (Alfenas $e t$ al. 1998). O padrão espacial em cada uma das freqüências alélicas foi então avaliado através de procedimentos de autocorrelação espacial (Sokal \& Oden 1978a, b, Diniz-Filho 1998).

A autocorrelação espacial em caracteres genéticos tem sido usualmente mensurada através do Índice I de Moran. $\mathrm{O}$ valor desse índice varia entre - $1,0 \mathrm{e}+1,0$ na maior parte dos casos, sendo estes valores seus máximos para autocorrelações negativas e positivas, respectivamente. Assim, sua interpretação é análoga à de um coeficiente de correlação, como o r de Pearson (Diniz-Filho 1998). Neste trabalho, cada frequiência alélica foi analisada através de um correlograma espacial, construído utilizando índices I de Moran estimados em 4 classes de distância espacial, com os seguintes limites superiores (em km): 54, 122, 190 e 235. Estas classes foram estabelecidas de modo a manter aproximadamente constante o número de subpopulações conectadas em cada uma delas, tornando assim os índices I de Moran que compõem o correlograma comparáveis, e com erros de estimativa semelhantes.

As inferências microevolutivas baseadas nos correlogramas só podem ser realizadas de forma efetiva quando diversos locos são examinados simultaneamente em diferentes subpopulações. Sokal \& Oden (1978a, b) denominam esta abordagem de multivariable, distinto de multivariate. A chave para essas inferências está na comparação dos diversos correlogramas entre si, na comparação das frequiências alélicas ("superfícies") e na associação dessas duas comparações (Diniz-Filho 1998). Os correlogramas foram comparados entre si através de distâncias Manhattan e estas foram, a seguir, interpretadas em função da correlação de Pearson entre as respectivas frequiências alélicas originais ao longo das subpopulações.

A fim de gerar uma hipótese nula da variação dos correlogramas sob um processo estocástico simples de divergência espacial entre as 10 subpopulações estudadas neste trabalho (em termos de tamanho amostral e distribuição espacial das subpopulações), a evolução estocástica das frequiências alélicas foi simulada utilizando a rotina PDSIMUL do programa PDAP (Phenotypic diversity analysis program - Jones et al. 1993). Essas simulações foram realizadas a partir de um agrupamento do tipo UPGMA (Sneath \& Sokal 1973) das distâncias geográficas, utilizado para representar na forma de um dendrograma (necessário para as simulações através do PDSIMUL) as distâncias entre as subpopulações.

Para as simulações, o modelo de evolução utilizado foi um processo estocástico do tipo Ornstein-Uhlenbeck (O-U) (Diniz-Filho 2000), que também gera uma relação exponencial entre divergência genética e distância geográfica, esperada tanto pela equação de Malecót-Morton para isolamento-por-distância quanto pelo modelo "stepping-stone".

As simulações foram iniciadas com frequiências alélicas de $0,5 \pm 0,04$, e como o PDAP foi originalmente desenvolvido para gerar variação em caracteres contínuos, foi utilizada também a opção "boundary" deste programa para restringir a variação das frequiências alélicas finais nas subpopulações entre zero e um. O valor da força de restrição foi dado por $1 / d_{\mathrm{MAX}}$, onde $d_{\mathrm{MAX}}$ é a maior distância geográfica entre as subpopulações (Diaz-Uriarte \& Garland 1996).

Foram geradas 500 distribuições das freqüências alélicas nas subpopulações, que foram então submetidas aos procedimentos de análise de autocorrelação espacial previamente descritos. Os correlogramas, assim calculados, permitiram conhecer a variação entre os perfis esperados sob um mesmo processo estocástico para 10 subpopulações com a mesma distribuição espacial das subpopulações de cagaiteira estudadas neste trabalho.

O SAAP 4.3 (Spatial Autocorrelation Analysis Program) (Wartenberg 1989) foi utilizado para as análises de autocorrelação espacial dos dados de isoenzimas. As análises de autocorrelação 
das freqüências alélicas obtidas nessas simulações foram realizadas utilizando uma versão modificada do programa IMCE (I de Moran e Correlogramas Espaciais) (Diniz-Filho 1993).

\section{Resultados}

Dos 140 índices I de Moran (27 frequiências em 4 classes de distância) obtidos na análise de autocorrelação espacial, 36 foram significativos ao nível de $5 \%$ de probabilidade (tabela 2). Utilizando o critério de Bonferroni, 15 correlogramas podem ser considerados significativos ao nível de $5 \%$ de probabilidade, ou seja, expressam um padrão espacial geral na variável.

Em geral, os correlogramas apresentaram índices I de Moran positivos e significativos na primeira classe de distância, associados a valores negativos e significativos na última classe, indicando um padrão clinal de variação espacial para o correlograma médio (figura 2). Assim,

Tabela 2. Índices I de Moran para quatro classes de distância entre as 10 subpopulações de cagaiteira. $O$ valor de $P$ indica a significância do correlograma como um todo, segundo o critério de Bonferroni. O $r$ é o coeficiente de correlação de Pearson entre os I de Moran e as classes de distância, para cada correlograma.

\begin{tabular}{|c|c|c|c|c|c|c|c|c|c|c|c|}
\hline \multirow{2}{*}{$\begin{array}{l}\text { Classe }(\mathrm{km}) \\
\text { Locos }\end{array}$} & \multirow[b]{2}{*}{ Alelos } & \multicolumn{2}{|l|}{54} & \multicolumn{2}{|l|}{122} & \multicolumn{2}{|l|}{190} & \multicolumn{2}{|l|}{235} & \multirow[t]{2}{*}{$P$} & \multirow[t]{2}{*}{$r$} \\
\hline & & & & & & & & & & & \\
\hline \multirow[t]{3}{*}{ SKDH } & 2 & 0,59 & $* *$ & 0,18 & & $-0,34$ & & $-0,81$ & $* *$ & 0,001 & $-0,990$ \\
\hline & 3 & 0,58 & $* *$ & 0,15 & & $-0,39$ & & $-0,74$ & $* *$ & 0,004 & $-1,000$ \\
\hline & 4 & 0,51 & $* *$ & 0,22 & & $-0,20$ & & $-0,91$ & $* *$ & 0,000 & $-0,960$ \\
\hline 6-PGD & 1 & $-0,16$ & & $-0,20$ & & $-0,34$ & & 0,23 & & 0,204 & 0,470 \\
\hline \multirow[t]{5}{*}{ EST-1 } & 1 & 0,31 & $*$ & 0,23 & & $-0,13$ & & $-0,80$ & $* *$ & 0,002 & $-0,910$ \\
\hline & 2 & 0,31 & $* *$ & 0,21 & $*$ & $-0,50$ & $*$ & $-0,44$ & $*$ & 0,031 & $-0,920$ \\
\hline & 3 & 0,50 & $* *$ & $-0,40$ & & $-0,67$ & $* *$ & 0,10 & & 0,023 & $-0,440$ \\
\hline & 4 & $-0,10$ & & 0,05 & & $-0,14$ & & $-0,25$ & & 0,656 & $-0,630$ \\
\hline & 5 & 0,58 & $* *$ & $-0,45$ & & 0,30 & $*$ & $-0,81$ & $* *$ & 0,002 & $-0,650$ \\
\hline \multirow[t]{5}{*}{ EST-2 } & 2 & 0,27 & $*$ & 0,17 & & $-0,05$ & & $-0,78$ & $* *$ & 0,000 & $-0,880$ \\
\hline & 3 & 0,29 & $*$ & $-0,40$ & & $-0,45$ & & 0,11 & & 0,193 & $-0,290$ \\
\hline & 4 & $-0,05$ & & $-0,29$ & & 0,20 & & $-0,29$ & & 0,353 & $-0,070$ \\
\hline & 5 & 0,30 & $*$ & $-0,51$ & $*$ & $-0,36$ & & 0,10 & & 0,188 & $-0,220$ \\
\hline & 6 & 0,18 & & 0,05 & & $-0,60$ & $*$ & $-0,07$ & & 0,054 & $-0,600$ \\
\hline \multirow[t]{5}{*}{ MDH } & 1 & 0,11 & & $-0,30$ & & $-0,26$ & & $-0,01$ & & 0,465 & $-0,280$ \\
\hline & 2 & 0,00 & & $-0,08$ & & $-0,46$ & & 0,07 & & 0,270 & $-0,180$ \\
\hline & 4 & $-0,24$ & & $-0,08$ & & $-0,12$ & & $-0,01$ & & 1,000 & 0,860 \\
\hline & 5 & $-0,08$ & & $-0,12$ & & $-0,35$ & & 0,08 & & 0,622 & 0,090 \\
\hline & 6 & 0,28 & $*$ & 0,19 & & $-0,53$ & $*$ & $-0,36$ & & 0,056 & $-0,880$ \\
\hline \multirow[t]{3}{*}{ PGI-2 } & 1 & 0,29 & $* *$ & 0,18 & $*$ & $-0,21$ & & $-0,64$ & $* *$ & 0,002 & $-0,950$ \\
\hline & 2 & 0,60 & $* *$ & $-0,02$ & & 0,04 & & $-0,99$ & $* *$ & 0,000 & $-0,890$ \\
\hline & 4 & 0,65 & $* *$ & 0,02 & & 0,00 & & $-1,03$ & $* *$ & 0,000 & $-0,910$ \\
\hline \multirow[t]{3}{*}{ PGM-1 } & 1 & 0,14 & & $-0,32$ & & 0,16 & & $-0,40$ & & 0,280 & $-0,450$ \\
\hline & 3 & 0,15 & & $-0,43$ & & 0,53 & $* *$ & $-0,64$ & $* *$ & 0,017 & $-0,280$ \\
\hline & 4 & 0,23 & & $-0,31$ & & 0,33 & $* *$ & $-0,64$ & $* *$ & 0,030 & $-0,500$ \\
\hline \multirow[t]{2}{*}{ PGM-2 } & 1 & $-0,08$ & & $-0,23$ & & $-0,30$ & & 0,14 & & 0,396 & 0,310 \\
\hline & 2 & $-0,14$ & & $-0,21$ & & $-0,04$ & & $-0,07$ & & 1,000 & 0,660 \\
\hline
\end{tabular}

$*$ - $\mathrm{P}<0,05 ; * *-\mathrm{P}<0,01$ 


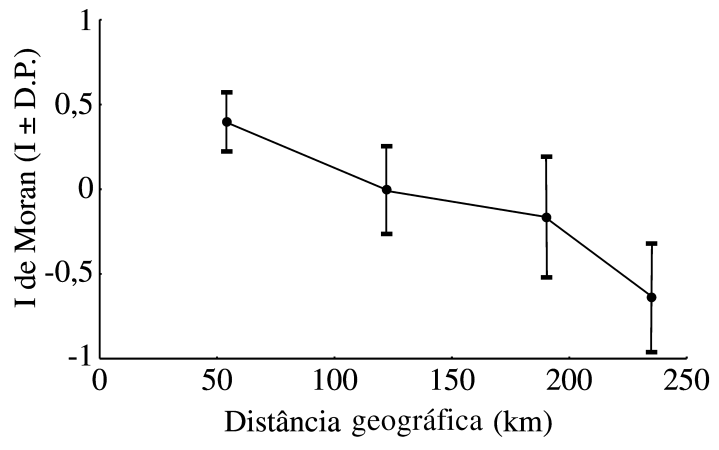

Figura 2. Correlograma médio para as freqüências alélicas de dez subpopulações de cagaiteira. As barras indicam um desvio-padrão em cada lado do I de Moran médio, em cada classe de distância.

subpopulações situadas a distâncias geográficas menores que $54 \mathrm{~km}$ tendem a ser similares para as frequiências alélicas, sendo que essa semelhança decai com o aumento da distância até que subpopulações situadas a mais de $200 \mathrm{~km}$ sejam completamente diferentes para essas frequiências. Esse padrão clinal é confirmado pelas correlações negativas e significativas entre o I de Moran e a classe de distância, para a maior parte dos alelos (tabela 2).

Correlogramas que apresentaram um padrão clinal de variação foram encontrados para os três alelos da enzima SKDH (figura 3A), para os alelos 1 e 2 da EST-1 (figura 3E), para o alelo 2 da EST-2 (figura 3G), e para os três alelos de PGI-2 (figura 3D). Padrões clinais nas primeiras classes de distâncias, mas seguidos de uma estabilização nas últimas classes, foram encontrados para o alelo $3 \mathrm{da}$ EST-1, para o alelo 6 da EST-2 e para o alelo 6 da MDH (figura 3B).

Padrões diferentes foram encontrados para alguns outros alelos analisados. No loco EST-1 (figura 3E), o correlograma do alelo 5 apresentou um coeficiente positivo nas primeiras classes, seguido de um decaimento e uma volta para um coeficiente positivo na terceira classe de distância. Para o primeiro loco da enzima PGM-1 (figura 3F), apenas os correlogramas dos alelos 3 e 4 foram significativos, mostrando um padrão semelhante ao apresentado pelo alelo 5 da EST-1, mas sem coeficientes significativos e positivos na primeira classe de distância.
Os valores encontrados para as distâncias Manhattan entre os correlogramas variaram entre 0,00 e 0,60 . A comparação destas distâncias com as correlações entre as freqüências alélicas originais mostrou um padrão no qual existe uma tendência de diminuição da similaridade entre os correlogramas correspondentes, à medida que decresce a correlação entre as frequiências (figura 4). Entretanto, há muitas freqüências pouco correlacionadas entre si que também apresentam correlogramas similares, gerando assim um padrão triangular de dispersão.

As simulações de evolução das freqüências alélicas sobre o espaço geográfico, utilizando um processo O-U no PDAP foram representativas, uma vez que a correlação cofenética do UPGMA da matriz de distâncias geográficas entre as subpopulações foi elevada $(\mathrm{rc}=0,96)$. Isso garante que a evolução das frequiências alélicas ao longo do dendrograma simula, de fato, um processo estocástico de difusão sobre o espaço geográfico.

De modo geral, os correlogramas obtidos a partir de dados simulados também apresentaram um padrão clinal de variação (figura 5A), sendo a maior parte das correlações entre o I de Moran e a classe de distância, negativa e significativa (figura 5B). É interessante notar que nos correlogramas simulados também aparecem padrões clinais com estabilização nas últimas classes de distância e também com um aumento dos I de Moran na terceira classe de distância, como encontrado para os correlogramas dos alelos 5 da EST-1 e 3 e 4 da PGM-1.

As distâncias Manhattan encontradas entre os correlogramas simulados, variaram entre $0,1 \mathrm{e} 1,0$, e estão relacionadas com as correlações entre as frequiências alélicas originais simuladas pelo padrão triangular de dispersão (figura 5C). Assim, as distâncias Manhattan encontradas entre os correlogramas reais estão dentro do intervalo de $95 \%$ das distâncias esperadas por um processo de evolução estocástica. Contudo, com o baixo número de subpopulações utilizadas, a faixa de variação dos perfis dos correlogramas obtidos por um processo estocástico é muito ampla e permite acomodar diferentes padrões, o que diminui o poder da simples inspeção desses perfis para rejeitar a hipótese de evolução neutra em consideração. 

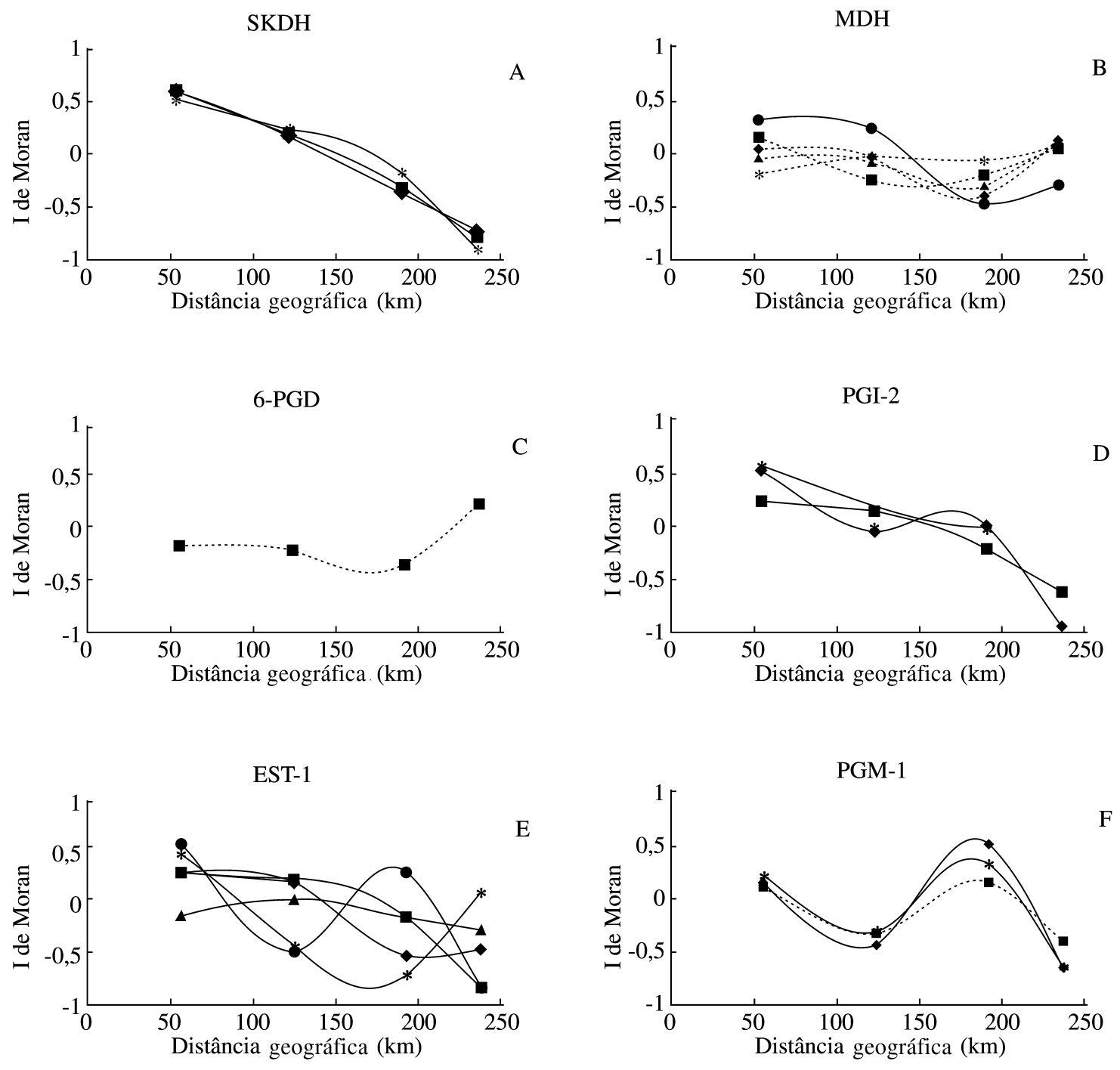

EST-2
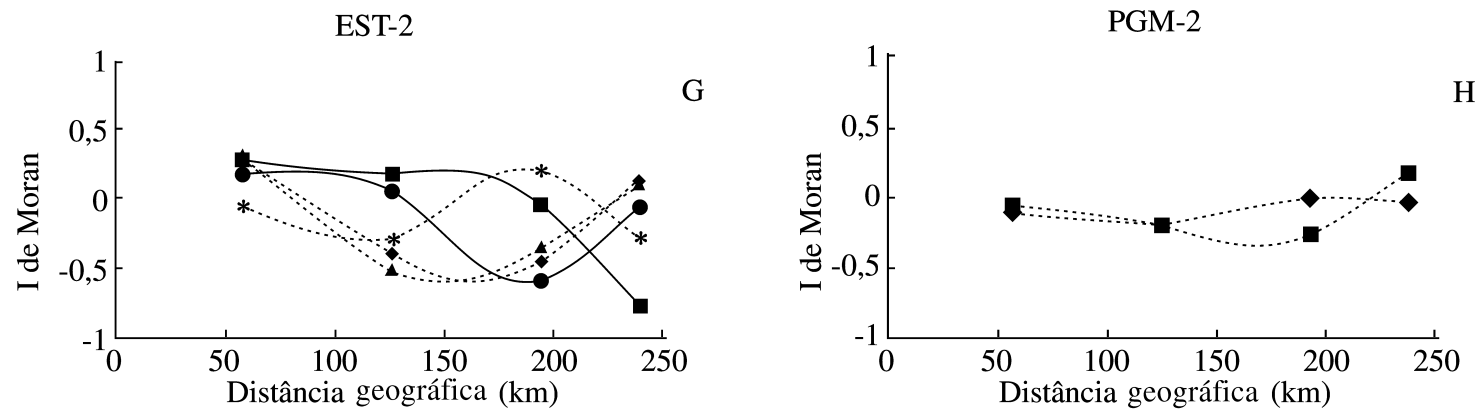

Figura 3. Correlogramas espaciais dos alelos para as dez subpopulações de cagaiteira nos diversos locos. A. SKDH; B. MDH; C. 6-PGD; D. PGI-2; E. EST-1; F. PGM-1; G. EST-2; H. PGM-2. A linha tracejada indica ausência de significância do correlograma, segundo o critério de Bonferroni e os símbolos $(\boldsymbol{\square}, \bullet, *, \mathbf{A}, \bullet)$ indicam os alelos em ordem crescente nos diferentes locos. 


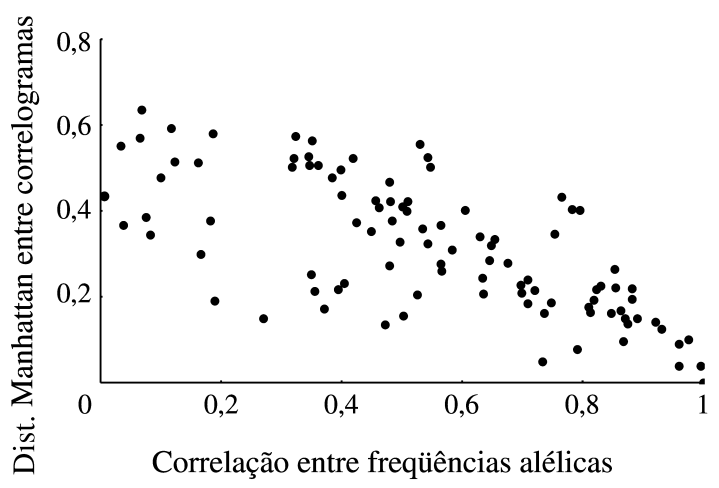

Figura 4. Relação entre a similaridade entre os correlogramas, medida pelas distâncias Manhattan, e a correlação de Pearson entre as frequiências alélicas.

\section{Discussão}

Uma correlação entre divergência genética e distância geográfica pode ser esperada sob diferentes modelos de evolução (Sokal \& Jacquez 1991). As populações podem divergir sob um processo estocástico simples (deriva genética), contrabalançado por fluxo gênico em curtas distâncias, o que gera um padrão conhecido como isolamento-por-distância (Wright 1943) ou "stepping-stone" (Kimura \& Weiss 1964), na dependência da espécie apresentar distribuição contínua ou discreta dos indivíduos na população. Outro modelo possível é aquele em que as pressões seletivas também estejam estruturadas no espaço geográfico gerando, conseqüentemente, estruturação espacial da divergência genética.

No caso de análises utilizando marcadores moleculares, as pressões de seleção devem ser consideradas como de baixa magnitude, não só pela natureza do marcador mas também pelo número elevado de variáveis (alelos) analisadas, que provavelmente não estão todas sob efeito do mesmo agente seletivo. É importante notar, entretanto, que no caso de marcadores isoenzimáticos ainda existem controvérsias sobre a neutralidade de alguns sistemas (Gray 1996).

Uma dificuldade quando se tenta inferir processos microevolutivos a partir de padrões de variação encontrados é justamente discernir entre os diversos processos microevolutivos possíveis que podem gerar o mesmo padrão espacial. A hipótese de evolução estocástica das freqüências alélicas
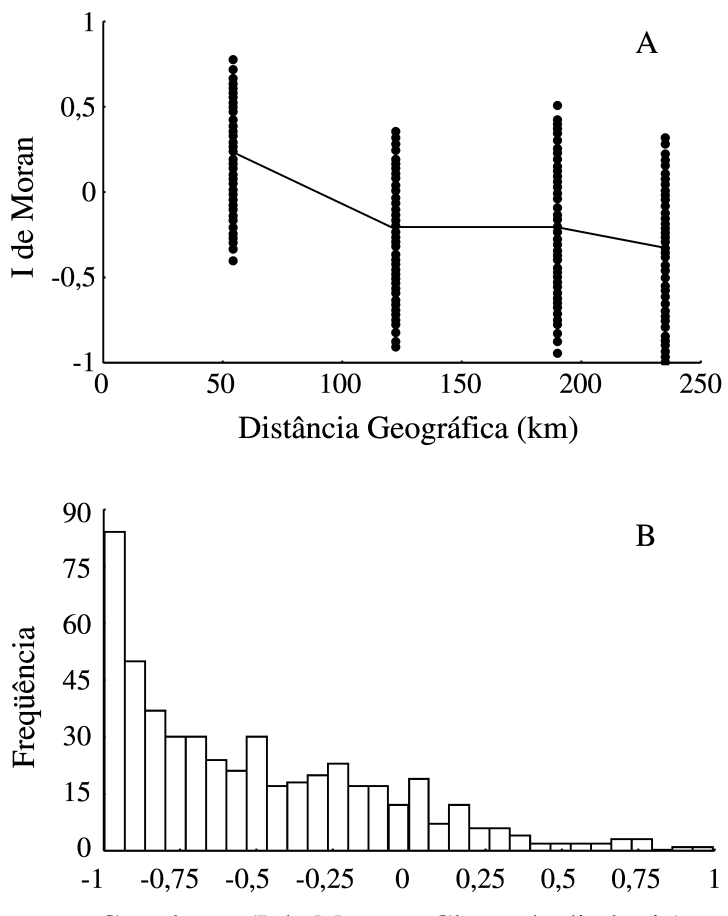

Correlação (I de Moran x Classe de distância)

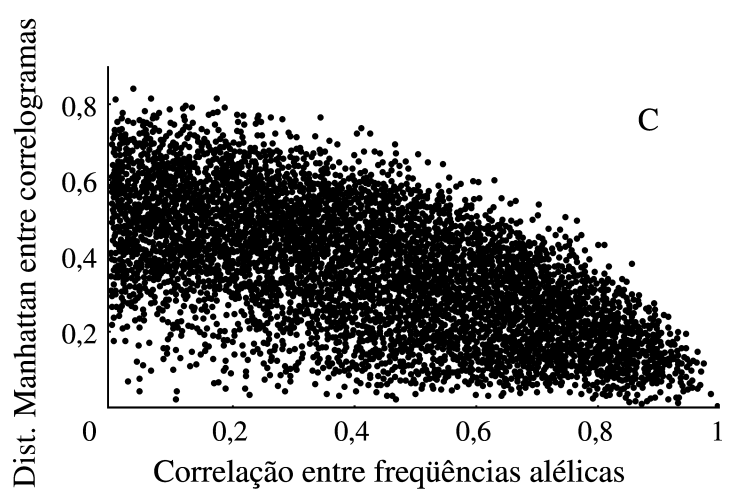

Figura 5. Resultados das análises de autocorrelação espacial das simulações de evolução estocástica de 500 frequêencias alélicas sobre o espaço geográfico, considerando uma relação exponencial entre divergência genética e distribuição espacial. A. Perfis dos correlogramas, sendo que a linha representa o correlograma médio; B. Distribuição de freqüência das correlações entre os I de Moran e a classe de distância geográfica, para cada um dos correlogramas simulados; C. Relação entre a similaridade entre os correlogramas simulados, medida pelas distâncias Manhattan, e a correlação de Pearson entre as freqüências alélicas.

pode ser apoiada pelas análises de autocorrelação espacial, que tem de fato auxiliado na tentativa de 
diferenciar estes processos microevolutivos (Sokal \& Jacquez 1991), baseadas nos padrões espaciais de cada uma das variáveis (freqüências alélicas). Um dos caminhos para isso é realizar a comparação entre os correlogramas através das distâncias Manhattan e relacioná-las com a correlação entre as frequiências alélicas originais. Esse procedimento é análogo ao teste de neutralidade de Lewontin-Krakauer, baseado na homogeneidade das estatísticas- $F$ (Lewontin \& Krakauer 1975).

Quando uma pressão seletiva está estruturada no espaço, são gerados correlogramas similares com padrões clinais de variação, porque a correlação entre as frequiências alélicas é alta. No entanto, em um processo evolutivo de divergência genética puramente estocástico, espera-se que os correlogramas sejam similares (distâncias Manhattan baixas), mostrando um padrão clinal de variação, mas apresentando freqüências alélicas não correlacionadas (Diniz-Filho 1998). Gera-se, desta forma, um padrão de dispersão triangular na relação entre as distâncias Manhattan e a correlação das freqüências alélicas originais.

Assim, padrões clinais de variação, associados ao padrão triangular de dispersão descrito anteriormente, são indicativos de processos estocásticos de divergência genética entre populações, tais como isolamento-por-distância ou "stepping-stone" (Sokal \& Jacquez 1991). Os correlogramas encontrados para a maior parte das freqüências alélicas neste trabalho são de fato clinais e mesmo alguns com perfis um pouco diferentes (como os alelos 3 e 4 da PGM-1 e 5 da EST-1) estão dentro da faixa de variação de perfis que podem ser obtidos em um processo estocástico de divergência genética, quando mensurado com base em apenas dez subpopulações, como mostrado pelas simulações (figura 5).

Assim, a importância das simulações está exatamente em estabelecer a diferença esperada entre os perfis de variação sob um processo estocástico de diferenciação e na definição da relação triangular. Nesse contexto, Sokal \& Jacquez (1991) sugere que distâncias Manhattan até 0,2 poderiam ser encontradas entre correlogramas gerados pelo mesmo processo em condições ideais de amostragem, embora esse valor possa ser bem maior quando poucas subpopulações são analisadas.
Isso é exatamente o que ocorre neste trabalho, onde distâncias Manhattan de até 0,6 foram encontradas entre os correlogramas de diferentes alelos. Entretanto, isso não reflete necessariamente diferenças de processos microevolutivos pois, nas simulações, distâncias Manhattan de até 1,0 foram encontradas utilizando a mesma configuração espacial de dez subpopulações sob um mesmo processo estocástico de diferenciação entre elas.

As análises realizadas permitiram avaliar os padrões espaciais de variação genética entre as subpopulações de cagaiteira e inferir que processos microevolutivos estão mais envolvidos nessa variação. Esses padrões podem ser melhor compreendidos quando se consideram, também, outros aspectos da biologia da planta, já que eles são determinados pela complexa interação que existe entre os fatores genéticos e ecológicos aos quais estes organismos estão submetidos. Os padrões espaciais de diferenciação observados nesse estudo são compatíveis com alguns aspectos da biologia da cagaiteira, tais como seu sistema de reprodução (misto) e o modo de polinização e dispersão dos frutos (Hamrick \& Loveless 1989). Entretanto, neste caso, mesmo a polinização e a dispersão por animais não devem ter um alcance muito grande, o que explica a elevada diferenciação entre as subpopulações. Isso, por sua vez, pode ser função de ação antrópica, já que a área estudada encontra-se fragmentada, não havendo, de modo geral, corredores ligando as subpopulações e que facilitem a movimentação desses dispersores.

Portanto, além dos próprios aspectos da biologia da espécie, um fator importante que deve ser levado em consideração para explicar os padrões espaciais encontrados neste estudo é o avançado processo de fragmentação, causado principalmente pela expansão da fronteira agrícola na região do Cerrado, que foi considerado recentemente como um dos "hotspots" mundiais (Myers et al. 2000). Esse processo reduz tanto o tamanho das subpopulações de cagaiteira quanto das de seus polinizadores e dispersores (aumentando a intensidade da deriva genética em cada uma delas) e deve estar contribuindo para a redução do alcance do fluxo gênico entre as subpopulações, conforme observado nesse estudo. Considerando que a cagaiteira é uma planta perene de ciclo longo (Donadio et al. 1992) e 
que esse processo de fragmentação é relativamente recente, espera-se que a divergência entre as subpopulações se torne ainda mais intensa no futuro.

O conhecimento do modo como a variabilidade genética está estruturada no espaço, aliado a outras características da espécie, pode fornecer subsídios para programas de conservação de germoplasma, auxiliando na definição de unidades evolutivamente estáveis (UEE) ou unidades de manejo (UM) (Eizirik 1996). Para as espécies que apresentam potencial de utilização econômico, estas informações também podem ser utilizadas em programas de domesticação e melhoramento genético das espécies (Gray 1996). Por exemplo, o intercepto dos perfis dos correlogramas é normalmente interpretado como sendo o raio médio de alcance do fluxo gênico, em termos de tempo ecológico, que restringe o efeito da evolução independente por deriva genética (Epperson 1997). Outro componente desse raio médio é a divergência (coalescência) ao longo do tempo histórico, que pode estar estruturada espacialmente (Avise 2000). Independente da sua interpretação biológica, nos correlogramas dos diversos alelos da cagaiteira, o intercepto "paramétrico" deve estar entre 60 e 120 km. Assim, a melhor estratégia de amostragem para a conservação da variabilidade genética total existente atualmente, seria a utilização do maior número possível de subpopulações, priorizando-se aquelas situadas entre si a uma distância maior que o alcance médio do fluxo gênico (no caso, distâncias maiores que $120 \mathrm{~km}$ ).

As análises realizadas sugerem que processos estocásticos, tais como o modelo de isolamento-por-distância ou "stepping-stone", são mais adequados para explicar o padrão espacial de divergência genética entre as subpopulações de cagaiteira na região sudeste do Estado de Goiás. Além disso, a existência de um padrão espacial na variação genética é importante para a compreensão de como ocorre a dinâmica dos alelos entre as subpopulações dessa espécie. Essa informação pode ser utilizada para otimizar a amostragem da variabilidade genética para sua subseqüente utilização.

Agradecimentos - Aos Drs. Roland Vencovsky, Divino Brandão e Fabrízio D'Ayala Valva pelas discussões e leitura de versões prévias do manuscrito. À Ludmila Ferreira Bandeira pela colaboração durante o trabalho. A realização deste trabalho só foi possível devido ao apoio financeiro dado à Escola de Agronomia e ao Instituto de Ciências Biológicas da Universidade Federal de Goiás, dentro do Programa Regional Integrado de Pesquisa e Extensão (PRIPE), pela FUNAPE/FINEP. Os autores agradecem também à Fundação de Apoio a Pesquisa (FUNAPE/UFG) pela bolsa de mestrado concedida a M.P.C. Telles e ao CNPq, pela bolsa de Produtividade em Pesquisa a J.A.F. Diniz-Filho.

\section{Referências bibliográficas}

ALFENAS, A.C., DUSI, A., ZERBINI-JÚNIOR, F.M., ROBINSON, I.P., MICALES, J.A., OLIVEIRA, J.R., DIAS, L.A.S., SCORTICHINI, M., BONDE, M.R., ALONSO, S.K., JUNGHANS, T.G. \& BRUNE, W. 1998. Eletroforese de isoenzimas e proteínas afins - fundamentos e aplicações em plantas e microrganismos. Universidade Federal de Viçosa, Viçosa.

ALFENAS, A.C., PETERS, I., BRUNE, W. \& PASSADOR, G.C. 1991. Eletroforese de proteínas e isoenzimas de fungos e essências florestais. Universidade Federal de Viçosa, Viçosa.

ALMEIDA, S.P. 1998. Frutas nativas do cerrado: caracterização físico-química e fonte potencial de nutrientes. In Cerrado: ambiente e flora. EMBRAPA-CPAC, Planaltina.

AVISE, J.C. 2000. Phylogeography. Harvard University Press, Cambridge.

BARBUJANI, G. 1987. Autocorrelation of gene frequencies under isolation-by-distance. Genetics 177:772-782.

COCKERHAM, C.C. 1969. Variance of gene frequencies. Evolution 23:72-84.

DIAZ-URIARTE, R. \& GARLAND, T. 1996. Testing hypotheses of correlated evolution using phylogenetically independent contrasts: sensitivity to deviations from Brownian motion. Systematic Biology 45:27-47.

DINIZ-FILHO, J.A.F. 1993. Spatial autocorrelation of morphometric variation in Lutosa brasiliensis (Orthoptera: Gryllacridoidea: Henicidae). Genetics and Molecular Biology 16:35-49.

DINIZ-FILHO, J.A.F. 1998. Análise de autocorrelação espacial: inferências microevolutivas e aplicações em genética de populações. Sociedade Brasileira de Genética, Ribeirão Preto. Série Monografias 6:82-145.

DINIZ-FILHO, J.A.F. 2000. Métodos filogenéticos comparativos. Holos, Ribeirão Preto. 120p.

DONADIO, L.C., MARTINS, A.B.G. \& VALENTE, J.P. 1992. Fruticultura tropical. FUNEP, Jaboticabal.

EIZIRIK, E. 1996. Ecologia molecular, genética da conservação e o conceito de unidades evolutivamente significativas. Brazilian Journal of Genetics 19:23-29.

EPPERSON, B.K. 1997. Gene dispersal and spatial genetic structure. Evolution 51:672-681.

FERREIRA, M.B. \& CUNHA, L.H.S. 1980. Dispersão de plantas lenhosas de cerrado - germinação e desenvolvimento. Informe Agropecuário 6:27-32.

FERREIRA, M.E. \& GRATTAPAGLIA, D. 1998. Introdução ao uso de marcadores moleculares em análise genética. EMBRAPA-CENARGEN, Brasília. 
GRAY, A. 1996. Genetic diversity and its conservation in natural populations of plants. Biodiversity Letters 3:71-80.

HAMRICK, J.L. \& LOVELESS, M.D. 1989. The genetic structure of tropical tree populations: associations with reproductive biology. In The evolutionary ecology of plants (J.H. Bock \& Y.B Linhart., eds.). Westview Press, Boulder, p.129-146.

HEYWOOD, J.S. 1991. Spatial analysis of genetic variation in plant populations. Annual Review of Ecology Systematic 22:335-355

JONES, J.A., DICKERMAN, A.W. \& GARLAND, T. 1993. PDAP - Phenotypic diversity analysis program. University of Wisconsin, Madison.

KIMURA, M. \& WEISS, G. 1964. The stepping stone model of population structure and the decrease of genetic correlation with distance. Genetics 49:561-576.

LEBRUN, P. \& CHEVALIER, M.H. 1988. Starch and polyacrylamid gel eletrophoresis of "Hevea brasiliensis": a laboratory manual. IRCA-CIRAD Publishers, Montpellier.

LEWONTIN, R.C. \& KRAKAUER, J. 1975. Testing the heterogeneity of F values. Genetics 80:397-398.

MYERS, N., MITTERMEIER, R.A., MITTERMEIER, C.G., FONSECA, G.A.B. \& KENT, J. 2000. Biodiversity hotspots for conservation priorities. Nature 403:853-858.

NEI, M. 1973. Analysis of genetic diversity in subdivided populations. Proceedings of National Academy of Sciences 70:3321-3323.

NEWTON, A.C., ALLNUTT, T.R., GILLIES, A.C.M., LOWE, A.J. \& ENNOS, R.A. 1999. Molecular phylogeography, intraspecific variation and the conservation of tree species. Trends in Ecology and Evolution 14:140-145.
PITEL, J.A. \& CHELIAK, W.M. 1984. Effect of extraction buffers on characterization of isoenzymes from vegetative tissues of five conifer species; a user's manual. Petawawa National Forestry Institute, Canadian Forestry Service, Petawawa.

PROENÇA, C.E.B. \& GIBBS, P.E. 1994. Reproductive biology of eight sympatric Myrtaceae from Central Brazil. New Phytologist 126:343-354.

REIS, M.S. 1996. Dinâmica da movimentação dos alelos: subsídios para conservação e manejo de populações naturais de plantas. Brazilian Journal of Genetics 19:37-47.

SLATKIN, M. \& ARTER, H.E. 1991. Spatial autocorrelation methods in population genetics. American Naturalist 138:499-517.

SNEATH, P.H. \& SOKAL, R.R. 1973. Numerical taxonomy: the principles and practice of numerical classification. W.H. Freeman, San Francisco.

SOKAL, R.R. \& JACQUEZ, G.M. 1991. Testing inferences about microevolutionary processes by means of spatial autocorrelation analysis. Evolution 45:152-168.

SOKAL, R.R. \& ODEN, N.L. 1978a. Spatial autocorrelation in biology. 1. Methodology. Biological Journal of Linnean Society 10:199-228.

SOKAL, R.R. \& ODEN, N.L. 1978b. Spatial autocorrelation in biology. 2. Some biological implications and four applications of evolutionary and ecological interest. Biological Journal of Linnean Society 10:229-249.

SOKAL, R.R. \& ODEN, N.L. 1991. Spatial autocorrelation analysis as an inferential tool in population genetics. American Naturalist 138:518-521.

WARTENBERG, D. 1989. SAAP: spatial autocorrelation analysis program. Exeter publishers, New York.

WRIGTH, S. 1943. Isolation by distance. Genetics 28:114-138.

WRIGTH, S. 1951. The genetical structure of populations. Annals of Eugenics 15:323-354. 